\title{
HOLOMORPHIC MARTINGALES AND INTERPOLATION BETWEEN HARDY SPACES: THE COMPLEX METHOD
}

\author{
P. F. X. MÜLLER
}

\begin{abstract}
A probabilistic proof is given to identify the complex interpolation space of $H^{1}(\mathbb{T})$ and $H^{\infty}(\mathbb{T})$ as $H^{p}(\mathbb{T})$.
\end{abstract}

\section{INTRODUCTION}

In this note, a soft propabilistic proof of P.W. Jones's theorem on the complex interpolation space between $H^{1}$ and $H^{\infty}$ is given. We shall work with N.Th. Varopoulos's space of holomorhpic martingales.

The observation presented in this article is that the use of a stopping time decomposition simplifies constructions of Serguei V. Kislyakov and Quanhua $\mathrm{Xu}$ to obtain the following

Theorem. The complex interpolation space

$$
\left[H^{1}(\mathbb{T}), H^{\infty}(\mathbb{T})\right]_{\Theta}, \quad 0<\theta<1,
$$

coincides with $H^{p}(\mathbb{T})$ provided that $\frac{1}{p}=1-\Theta$.

As is well known this result has been obtained by P.W. Jones using $L^{\infty}$ estimates to the $\bar{\partial}$ problem (see $[\mathrm{J}]$ ). His work also contains the description of the real interpolation spaces for the couple $\left(H^{1}, H^{\infty}\right)$. At about the same time Jean Bourgain obtained a Marcinkiewicz type decomposition, using completely different techniques (see [B1]). Recently in a series of papers Jean Bourgain [B2], Serguei Kislyakov [K1], [K2], [K-X], Gilles Pisier [P] and Quanhua Xu [X1], [X2] obtained deep results concerning real and complex interpolation methods between vector-valued, weighted Hardy spaces of analytic functions. S. Kislyakov's paper [K1] contains the following idea to approximate the characteristic function $\mathbf{1}_{\{|f|<\lambda\}}$ by analytic functions on the circle $\mathbb{T}:$ He starts

$$
\alpha=\max \left\{1, \frac{|f|}{\lambda}\right\}
$$

and then considers

$$
\varphi=\frac{1}{\alpha+i H \alpha}
$$

where $H$ denotes the Hilbert transform on $\mathbb{T}$.

Received by the editors August 11, 1992 and, in revised form, February 1, 1994; originally communicated to the Proceedings of the AMS by Dale Alspach.

1991 Mathematics Subject Classification. Primary 60G46, 42B30.

Key words and phrases. Holomorphic martingales, complex interpolation method, Hardy spaces. 
In [K2] S. Kislyakov uses these approximations and constructs an "analytic partition of unity", thereby proving J. Bourgain's theorem on absolutely summing operators on the disc algebra.

Then $\mathrm{Q}$. $\mathrm{Xu}$, using this analytic partition of unity, was able to give an elementary proof of P.W. Jones's complex interpolation theorem (see [X1]).

The use of probability allows us to simplify Q. Xu's proof further: We start with a stopping-time argument which gives a decomposition of a given element $f \in H^{p}(\Omega)$ into a sum of functions $d_{i} \in H^{\infty}(\Omega)$ in such a way that we have good control over the supports and $H^{\infty}(\Omega)$ norms (of the functions $d_{i}$ ). Then one defines the vector-valued analytic functions on the strip $S=\{\xi: 0<\operatorname{Re} \xi<$ $1\}$, which is required to conclude that

$$
H^{p}(\Omega) \subseteq\left[H^{1}(\Omega), H^{\infty}(\Omega)\right]_{\Theta}
$$

(for a definition of the complex interpolation method see [B-L] or [Ja-Jo]).

These notes are also a continuation of [M] where a probabilistic argument was given to identify the real interpolation spaces between $H^{\infty}(\mathbb{T})$ and $H^{1}(\mathbb{T})$. For sake of completeness let us recall the concept of holomorphic martingales: $\left(z_{t}\right)_{t \geq 0}$ denotes the complex Brownian motion on the Wiener space $(\Omega,(\mathscr{F}), \mathscr{F}, P)$.

Definition. A random variable $X: \Omega \rightarrow \mathrm{C}$ is called holomorphic if and only if the conditional expectations

$$
X_{t}=\mathbf{E}\left(X \mid \mathscr{F}_{t}\right)
$$

admit a stochastic integral representation of the form

$$
X_{t}=X_{0}+\int_{0}^{t} f_{s} d z_{s}
$$

where $f_{s}: \Omega \rightarrow \mathbf{C}$ is measurable with respect to $\mathscr{F}$.

Accordingly $H^{p}(\Omega)$ denotes the subspace of $L^{p}(\Omega)$ which consists of holomorphic random variables. On a general $\mathscr{F}$ martingale with stochastic integral representation

$$
Y_{t}=Y_{0}+\int_{0}^{t} f_{s} d x_{s}+\int_{0}^{t} g_{s} d y_{s}
$$

we define

$$
(\mathscr{H} Y)_{t}=-\int_{0}^{t} g_{s} d x_{s}+\int_{0}^{t} f_{s} d y_{s}
$$

This martingale transform is called the stochastic Hilbert transform, because for $Y \in L^{2}(\Omega)$ one obtains $Y+i \mathscr{H} Y \in H^{2}(\Omega)$.

Being a martingale transform, $\mathscr{H}$ defines a bounded operator on $L^{p}(\Omega)$ for $1<p<\infty$. The corresponding norms are denoted by $N_{p}$. For a function $f \in L^{p}(\Omega)$ we denote by $f^{*}$ it's martingale maximal function, i.e.

$$
f^{*}:=\sup _{s}\left|\mathbf{E}\left(f \mid \mathscr{F}_{s}\right)\right| \text {. }
$$

For $L^{p}(\Omega), 1<p \leq \infty$, we have $\|f\|_{p} \leq M_{p}\left\|f^{*}\right\|_{p}$. We shall write $C_{p}$ for the larger of $N_{p}$ and $M_{p}$. Below we shall give a proof of 
Theorem 1. The complex interpolation space $\left[H^{1}(\Omega), H^{\infty}(\Omega)\right]_{\Theta}, 0<\Theta<1$, coincides with $H^{p}(\Omega)$ provided that $\frac{1}{p}=1-\Theta$.

As shown by $\mathrm{N}$. Th. Varopoulos [V], there exist operators $M, N$ such that for $1 \leq p \leq \infty$ the diagram

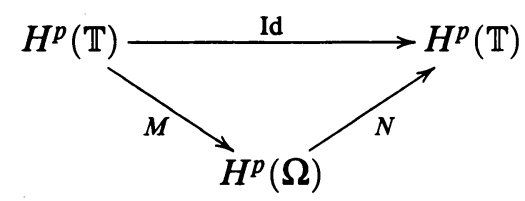

commutes. We thus derive Jones's theorem from Theorem 1.

THE BASIC DECOMPOSITION

In this section, we use a sequence of stopping times to decompose a given $f \in H^{p}(\Omega)$ into a $p$-absolutely convergent series of functions $d_{i} \in H^{\infty}(\Omega)$.

Take $f \in H^{p}(\Omega), 1<p<\infty$. Let

$$
m_{1}:=\inf \left\{n: P\left\{f^{*}>2^{n}\right\} \leq \frac{1}{4}\right\} .
$$

Having defined $m_{1}, \ldots, m_{j-1}$ we put

$$
E_{j-1}=\left\{f^{*}>2^{m_{j-1}}\right\}
$$

and define

$$
m_{j}:=\inf \left\{n>m_{j-1}: P\left\{f^{*}>2^{n}\right\} \leq \frac{1}{4} P\left(E_{j-1}\right)\right\} .
$$

Using this sequence of integers we define natural stopping times as

$$
\tau_{0}(\omega):=0, \quad \tau_{j}(\omega):=\inf \left\{t \in \mathbf{R}:\left|\mathbf{E}\left(f \mid \mathscr{F}_{t}\right)(\omega)\right| \geq 2^{m_{j}}\right\} .
$$

These stopping times are used to chop off the large parts of $f$. We let $\mathscr{F}_{\tau_{j}}$ be the $\sigma$-algebra generated by the stopping time $\tau_{j}$, and we put $f_{j}:=\mathbf{E}\left(f \mid \mathscr{F}_{\tau_{j}}\right)$. Finally we form the martingale differences $d_{j}=f_{j+1}-f_{j}$.

It is easily observed that $d_{j}$ lies in $H^{\infty}(\Omega)$, has its support contained in $E_{j}$, and is dominated by $2^{m_{j+1}}$ and that

$$
f-\mathbf{E}(f)=\sum_{j=0}^{\infty} d_{j} .
$$

For our purposes we need to improve this decomposition.

We let

$$
\alpha_{j}(\omega)=\max \left(1, \delta \frac{f^{*}}{2^{m_{j}}}\right) .
$$

The function

satisfies the following conditions:

$$
\varphi_{j}=\frac{1}{\alpha_{j}+i \mathscr{H} \alpha_{j}}
$$

(i) $\varphi_{j} \in H^{\infty}(\Omega)$ with $\left\|\varphi_{j}\right\|_{\infty} \leq 1$.

(ii) For $k \geq j+1$ and $\omega \in E_{k}$ we obtain the pointwise estimate

$$
\left|\varphi_{j}(\omega)\right| \leq \frac{1}{\delta}\left(\frac{2^{m_{j+1}}}{2^{m_{k}}}\right) .
$$


(iii)

$$
\begin{aligned}
\int_{\Omega}\left|1-\varphi_{j}\right|^{p} d P & \leq C_{p}^{p} \int_{\Omega}\left|1-\alpha_{j}\right|^{p} d P \\
& \leq \delta^{p} C_{p}^{p} \int_{E_{j+1}}\left(\frac{f^{*}}{2^{m_{j+1}}}\right)^{p} d P .
\end{aligned}
$$

Subsequently we fix $\delta$ so that $|\log \delta|^{1 / p} \delta C_{p}^{2} \leq \frac{1}{64}$.

\section{THE PROOF OF THEOREM 1}

Given $1<p<\infty, \Theta:=1-1 / p$ and $f \in H^{p}(\Omega)$. In this section we shall construct an analytic function $F$ on the strip $S=\{\zeta=\eta+i \xi: 0 \leq \eta \leq 1\}$ which satisfies

(1) $\|F(\Theta)-f\|_{H^{p}(\Omega)} \leq \frac{1}{4}\|f\|_{H^{p}(\Omega)}$,

(2) $\sup _{\xi \in \mathbf{R}}\|F(i \xi)\|_{H^{1}(\Omega)} \leq C\|f\|_{H^{p}(\Omega)}^{p}$,

(3) $\sup _{\xi \in \mathbf{R}}\|F(1+i \xi)\|_{H^{\infty}(\Omega)} \leq C$.

These three properties of $F$ imply that $H^{p}(\Omega) \subset\left[H^{1}(\Omega), H^{\infty}(\Omega)\right]_{\Theta}$, which is enough to identify the complex interpolation space $\left[H^{1}(\Omega), H^{\infty}(\Omega)\right]_{\Theta}$ with $H^{p}(\Omega)$ (see [Ja-Jo]).

We start with the basic decomposition

$$
f=\sum_{i=0}^{\infty} d_{i}
$$

construct $\varphi_{i}$ as in $\S 2$, and define

$$
F(\xi)=\sum_{j=0}^{\infty} d_{j} \varphi_{j} 2^{(p(1-\xi)-1) m_{j+1}} .
$$

As $F(\boldsymbol{\Theta})-f=\sum_{j=0}^{\infty} d_{j}\left(1-\varphi_{j}\right)$, we have to check the following three inequalities: For all $\xi \in \mathbf{R}$

(1) $\left\|\sum_{j=0}^{\infty} d_{j}\left(1-\varphi_{j}\right)\right\|_{L^{p}(\Omega)} \leq \frac{1}{4}\|f\|_{L^{p}(\Omega)}$,

(2) $\left\|\sum_{j=0}^{\infty} d_{j} \varphi_{j} 2^{p(1-i \xi-1) m_{j+1}}\right\|_{L^{1}(\Omega)} \leq C\|f\|_{L^{p}(\Omega)}^{p}$,

(3) $\left\|\sum_{j=0}^{\infty} d_{j} \varphi_{j} 2^{p i \xi} 2^{-m_{j+1}}\right\|_{L^{\infty}(\Omega)} \leq C \delta^{-1}$.

Verification of (1). The important idea to change the order of summation below is taken from S. Kislyakov's paper [K2].

The choice of the sequence $\left(m_{j}\right)$ implies that the $p$ th power of the left-hand side of (1) is dominated by

$$
|\log \delta| \sum_{j=0}^{\infty} \int\left|d_{j}\left(1-\varphi_{j}\right)\right|^{p} d P
$$

Using the $L^{\infty}$ estimates for $d_{j}$ and the $L^{p}$ boundedness of the stochastic Hilbert's transform we estimate the above sum by

$$
|\log \delta| C_{p} \sum_{j=0}^{\infty} 2^{m_{j+1} p} \int_{\Omega}\left|1-\alpha_{j}\right|^{p} d P
$$


Above we obtained

$$
\int_{\Omega}\left|1-\alpha_{j}\right|^{p} \leq \delta^{p} 2^{-\left(m_{j+1}\right) p} \sum_{k=j+1}^{\infty} 2^{\left(m_{k+1}\right) p} P\left(E_{k}\right) .
$$

Using this estimate and changing the order of summation we can dominate the left-hand side of (1) by

$$
\begin{aligned}
|\log \delta| \delta^{p} C_{p}^{p} \sum_{k=1}^{\infty} P\left(E_{k}\right) 2^{m_{k+1} p} \sum_{j=0}^{k} 2^{m_{j+1} p} 2^{-m_{k+1} p} & \leq|\log \delta| \delta^{p} 16 C_{p}^{p} \sum_{k=1}^{\infty} P\left(E_{k}\right) 2^{m_{k+1} p} \\
\leq|\log \delta| \delta^{p} 16 C_{p}^{p} \int_{\Omega} f^{* p} d P & \leq|\log \delta| \delta^{p} 16 C_{p}^{2 p} \int_{\Omega}|f|^{p} d P
\end{aligned}
$$

Verification of (2). The left-hand side of (2) is clearly dominated by

$$
\sum_{i=0}^{\infty} \int\left|d_{i}\right| \varphi_{i} 2^{(p-1) m_{i+1}} d P
$$

Using $L^{\infty}$ estimates of $d_{i}$ and the fact that $d_{i}$ is supported in the set $\left\{f^{*}>\right.$ $2^{m_{i}}$ \} we can dominate the above sum by

$$
\sum_{j=0}^{\infty} 2^{m_{j+1} p} P\left\{f^{*}>2^{m_{j}}\right\} .
$$

The choice of $\left(m_{j}\right)$ allows one to dominate this sum by

$$
2 \sum_{j=0}^{\infty} 2^{m_{j+1} p} P\left\{f^{*}>2^{m_{j+1}-1}\right\}
$$

which is bounded by $4\left\|f^{*}\right\|_{L^{p}(\Omega)}^{p}$.

Verification of (3). The $L^{\infty}$ bounds on $d_{j}$ and the fact that supp $d_{j} \subseteq E_{j}$ gives an estimate for the left-hand side of (3) by

$$
\delta^{-1}\left\|\sum_{j=0}^{\infty} \mathbf{1}_{E_{j}}\left|\varphi_{j}\right|\right\|_{\infty}
$$

By construction the above sum can be dominated by

$$
\delta^{-1}\left\|\sum_{j=0}^{\infty} \mathbf{1}_{E_{j} \backslash E_{j+1}} 2^{-m_{j}}\left(\sum_{l=0}^{j} 2^{+m_{l}}\right)\right\|_{\infty},
$$

which is bounded independently of $f$.

\section{REFERENCES}

[B-L] J. Berg and J. Löfström, Interpolation spaces, Springer Verlag, New York, 1976.

[B1] J. Bourgain J., New Banach space properties of the disc algebra and $H^{\infty}$, Acta Math. 152 (1984), 1-48.

[B2] _ Some consequences of Pisier's approach to interpolations, preprint 1991. 
[D] R. Durrett, Brownian motion and martingales in analysis, Wadsworth, 1984.

[J] P. W. Jones, $L^{\infty}$ estimates for the $\partial$ problem in a half space, Acta Math. 150 (1983), 37-152.

[Ja-Jo] S. Janson and P. W. Jones, Interpolation between $H^{p}$ spaces, J. Funct. Analysis 48 (1982), 58-80.

[K1] S. V. Kislyakov, Truncating functions in weighted $H^{p}$ and two theorems of $J$. Bourgain, Uppsala University, Dept. of Math., Report 1989:10.

[K2] - Absolutely summing operators on the disc algebra, Analysis and Algebra 3 (1991), 1-77; English transl., St. Petersburg Math. J. 3 (1992), 705-774..

[K-X] S. V. Kislyakov and Q, Xu, Interpolation of weighted and vector valued Hardy spaces, Publ. Inst. Recherche. Math. Lille 25 (1991).

[M] P. F. X. Müller, Holomorphic Martingales and interpolation between Hardy spaces, J.'Analyse Math. 61 (1993), 327-337.

[P] G. Pisier G, Interpolation between $H^{p}$ spaces and non commutative generalization, Pacific J. Math. 155 (1992), 341-368.

[V] N. Th. Varopoulos, The Helson-Szegö theorem and $A_{p}$ functions for Brownian motion and several variables, J. Funct. Anal. 39 (1980), 85-121.

[X1] Q. Xu, Elementary proofs of two theorems of P.W. Jones on interpolation between Hardy spaces, Ann. Inst. Fourier (Grenoble) 42 (1992), 875-889.

[X2] _ Notes on interpolation of Hardy spaces, preprint 1990.

InSTITUt FÜr MAThematik, J. KePler Universität LinZ, A-4040 LinZ, AUSTRIA

E-mail address: paul.mueller@jk.uni-linz.ac.at 